\title{
Restricted posterior cortical lesion influences on learning and retention of a successive brightness discrimination
}

\author{
F. ROBERT TREICHLER, MELICENT J. HUBBARD and ELIZABETH J. HOLLAND \\ Kent State University, Kent, Ohio 44240
}

\begin{abstract}
Rats with lesions of the occipital pole or a more anterior extrastriate area were tested on either original learning of a successive brightness discrimination or retention of this task after acquisition as a normal. Equivalent original learning losses relative to normals were sustained from both lesions, but while extrastriates showed substantial savings in retention, rats with occipital pole damage required as many relearning trials as were necessary for original learning by brain-damaged groups. It was suggested that the effect of the anterior extrastriate lesion was to impair a spatial or positional component of discrimination performance, and that this component might differ from one related to visual task properties, especially in terms of recovery of function following brain damage.
\end{abstract}

An issue of concern in the investigation of central nervous mechanisms related to brightness discrimination has been the possibility that the infliction of localized cortical damage may not only render a rat deficient in visual capability, but may also modify its preferential selection of simultaneously presented discriminanda. Posterior cortical lesions have been noted by Altman (1962) and by Stratton and Edwards (1971) to reduce the likelihood of choice of an environment with a low illumination level. If differentially illuminated escape doors, rather than environments, provide the discriminanda in a Yerkes apparatus, Parker, Erickson, and Treichler (1969) have shown that the usual extensive posterior lesion attenuates repetitive positional choice and produces a slight, but significant, tendency for selecting a brighter alternative. A more recent experiment by Parker and Treichler (1973) has indicated that these two preference phenomena are associated with unique and different cortical areas. According to their report, reduced preferences for dark environments are related to removal of the most posterior striate areas, while reduction in positional responding and increased choice of a brighter escape door accompanies the loss of more anterior extrastriate areas. They contended that the commonly administered large posterior lesion induces behavioral consequences which relate to both of these zones. These investigators have also indicated that the positional preference phenomenon bears little relationship to actual performance on a simultaneous brightness discrimination presented in a Yerkes apparatus, since rats with a restricted anterior extrastriate lesion showed no learning

Ms. Holland's contributions were slapinrted under terms of NSF Undergraduate Participation Award, GY-6160. Nuw at Kansas State University.

Requesty for reprints should be addressed to $F$. Robert Treichler, Department of Psychology, Kent State University, Kent, Uhio 44240 . impairment relative to normals on this task, while animals with removals limited to more posterior polar areas were markedly impaired.

The outcomes of these investigations of simultaneous discrimination (i.e., with both discriminanda present on each trial) raise some questions about the nature of the consequences of similar lesions upon successive discriminations. The classic experiment employing the latter evaluative instrument has been reported by Thompson and Malin (1961). In this study, three groups of rats learned a successive task which entailed intratrial presentation of identical stimuli with intertrial variation of discriminanda associated with the position of a correct escape door. One group was maintained as a normal control and the other two provided differential lesion locus conditions. All were subsequently tested for retention in relearning. Damage was always inflicted between acquisition and relearning, and, while one group received a posterior visual lesion, the other sustained damage to a slightly larger anterior area which extended forward to the frontal pole. Although direct comparisons of the behavioral consequences of the two lesions were not undertaken, both kinds of damage yielded retention deficits as indicated by error savings scores relative to the controls. Thompson and Malin (1961) reported that both loci influenced performance on the successive task because both areas "might be essential for the process of compounding visual and spatial stimuli." Because of a subsequent experiment in which simple spatial retention was selectively impaired by their anterior lesion, they considered this cortical area to be a specific contributor to the spatial (positional) component of performance.

When the effects of Parker and Treichler's (1973) anterior extrastriate damage upon positional preference and Thompson and Malin's (1961) anterior removal influences on successive discrimination are considered 


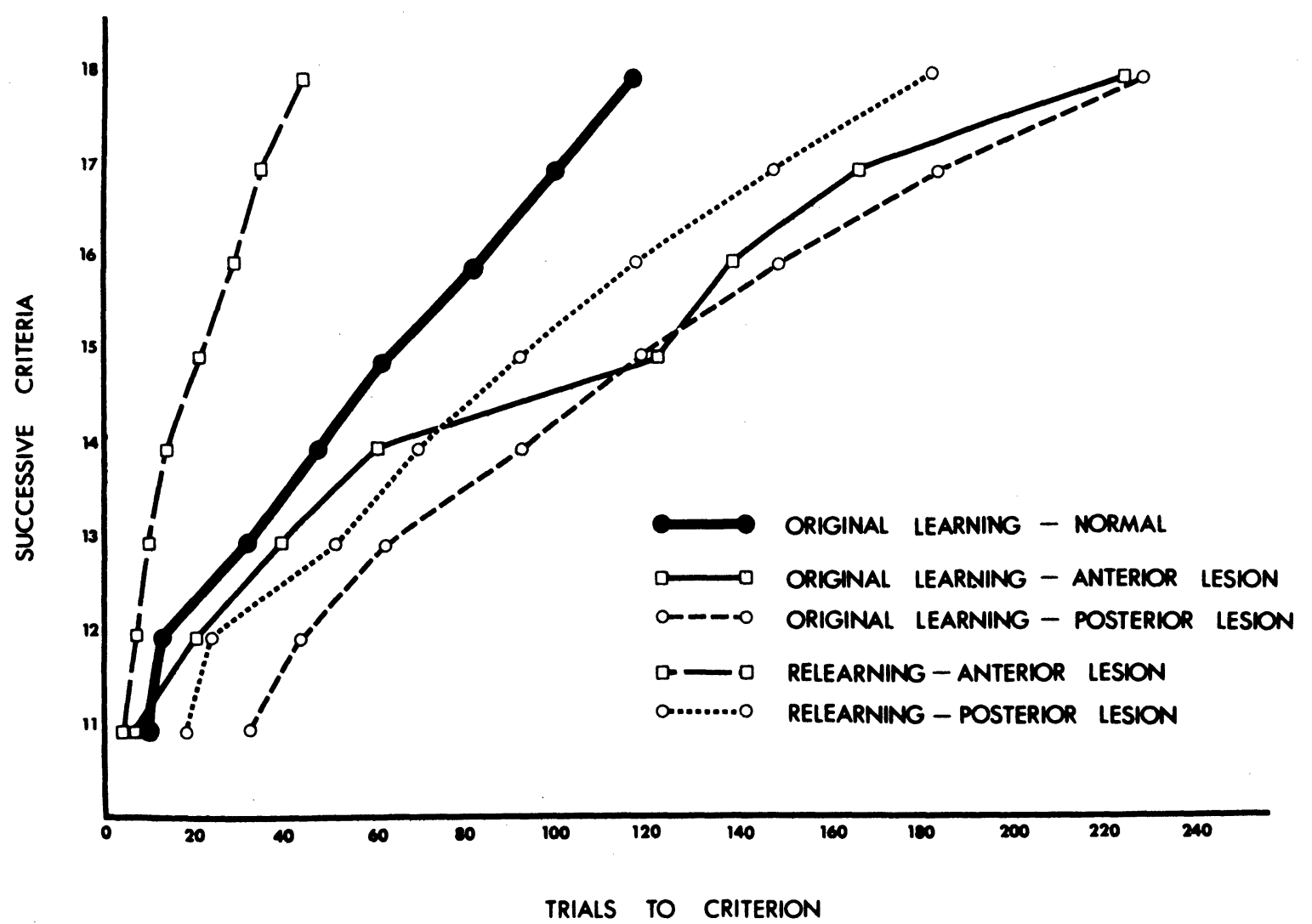

Figure 1. Mean numbers of trials required to attain successively more stringent criteria by the various treatment groups. (Normal $N=20$, all other groups $N=10$ ).

concurrently, some parallels are suggested. One possibility is that the more anterior lesions in both investigations affected a common spatial factor, and this damage caused the retention deficit on the successive problem. The present investigation attempted to provide a test of this interpretation by determining whether or not extrastriate and occipital-pole removals like those of Parker and Treichler (1973) influenced performance on a successive brightness discrimination. Additionally, the experiment was designed so that both original learning and retention of the successive task could serve as evaluative instruments for both the variously brain-damaged groups and a group of normal controls.

\section{METHOD}

Forty experimentally naive male Long-Evans rats, aged 80-90 days, served as subjects. Original learning was provided for 20 normal animals and to two groups of 10 lesioned animals. After original learning, the normals were randomly assigned to one of two groups receiving lesions and were subsequently tested for postoperative relearning. All animals underwent a recovery period of at least 2 weeks after surgery.

The two lesion conditions provided in the present study were identical to the groups designated "anterior" and "posterior" by Parker and Treichler (1973; see their Figure 5). These areas were defined by intended bilateral aspirative removal of either the rostral or caudal half of that neocortical zone extending from beneath bregma to the tip of the occipital pole and from the midline to the rhinal fissure.

Testing was conducted in a Yerkes apparatus, with translucent doors, in which momentary scrambled and graded footshock could be administered by the experimenter. After each rat was placed in the apparatus and allowed to explore with all doors open for $10 \mathrm{~min}$, it was given six trials on which escape was provided when a $7.62-\mathrm{cm}$-wide floor treadle in front of either exit door was depressed. On the following day, each animal was given 30 discrete correctional trials on which equal numbers of exits via the right and left doors were enforced according to a counterbalanced sequence. Training on the successive task began on the following day and required a different positional choice (right or left) when bright or dim stimuli were presented on the escape doors. Stimulus values were .343 nits and 548.8 nits for the dim and bright doors, respectively, when measured via a McBeth illuminometer placed at the nearest possible choicepoint. The assignment of particular cues to correct positions was counterbalanced within each group. Thirty noncorrectional escape/avoidance trials were presented each day at approximately $15-\mathrm{sec}$ intertrial intervals, and testing was continued until each subject had attained a criterion of 18 correct in 20 consecutive responses or had completed 300 trials.

\section{RESULTS}

Figure 1 displays the mean number of trials to attain 
successively more stringent criteria for the normal and the variously lesioned groups. Nonparametric tests were applied to the scores derived from the number of trials to reach the 18 of 20 criterion. Wilcoxen tests indicated that the original learning of anterior and posterior brain-damaged animals did not differ significantly. However, in relation to acquisition by normals, each of these groups was significantly impaired $(p<.05)$. Application of Mann-Whitney tests revealed that relearning by posterior animals was impaired when compared to normals, but was not different from the original learning of either ablated group. Contrastingly, the anterior extrastriate group exhibited significant $(p<.05)$ savings in relearning relative to normal acquisition. An examination of error patterns indicated no obvious trend in visual or spatial preferences which might have been systematically related to effects of the lesion.

Histological examination of the extents and locations of cortical surface removal and subcortical degeneration are indicated in Figure 2, and consequences appear comparable to those seen in the lesions inflicted by Parker and Treichler (1973). Degeneration of dorsal lateral geniculate nuclei occurred as a result of the posterior lesion while the anterior lesion resulted in focal degeneration of nuclei ventralis thalami. Specific comparison of cortical involvement and thalamic degeneration in the groups which received brain damage before or after original learning revealed no differential lesion placement or extent other than as a consequence of assignment to anterior or posterior groups.

\section{DISCUSSION}

The outcomes displayed in Figure 1 and their accompanying tests of significance reveal a fairly straightforward set of consequences from the brain damage inflicted in this experiment. Both of the lesions could impair successive task performance, but, unlike Thompson and Malin's (1951) finding in this regard, the present losses were specific to the original learning phase of testing. When tested on retention of the successive problem, animals with damage limited to the occipital pole were impaired relative to normals but were not different from those groups which had received damage prior to their original learning. However, the retention performance of rats with the present restricted anterior lesion revealed substantial savings.

The difference between the present retention results and those of Thompson and Malin (1951) could be ascribed to the inclusion of a rather large frontal zone in the latter experiment. Thus, it seems that, as Thompson and Malin (1951) suggested, spatial factors may contribute to performance on the kind of conditional problem which comprises a successive brightness discrimination, but the area specific to this phenomenon

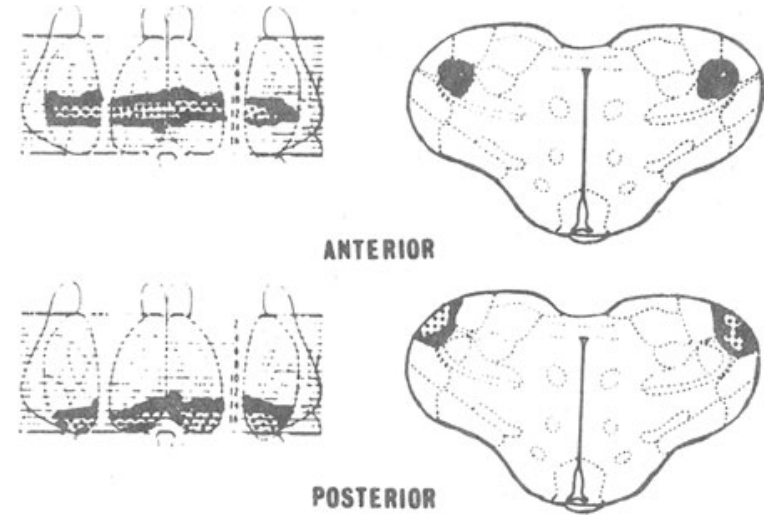

Figure 2. Extent of cortical lesions (left) and thalamic degeneration (right) for rats with anterior or posterior removals. Stippled areas indicate the subject with smallest zone of involvement and black areas indicate the animal with the largest.

may not include all that neocortex anterior to the striate zone. Rather, a fairly limited extrastriate area could be responsible, and the effect of other cortical involvement may be to impair some behavioral characteristics which contribute to retention test performances in much the same manner as Horel, Bettinger, Royce, and Meyer (1966) have shown when assessing anterior lesion influences on simultaneous brightness discrimination. This interpretation is also supported by Parker and Treichler's (1973) finding of no impairment in the simultaneous task when damage is limited to the extrastriate "anterior" area. Of course, these are matters of speculation at present, but such possibilities do provide questions for further empirical test.

Another outcome from this experiment which seems to warrant further investigation is the consequence of successive discrimination training upon retention capabilities of rats that subsequently undergo the limited anterior lesion. Because this lesion seems to induce a significant loss in acquisition while substantial savings are noted in the retention of similarly brain-damaged animals, it is possible that this result represents an example of protection from impairment by prior experience. As such, this task and lesion locus seem to offer a potentially important test situation for some of the theoretical explanations which have been advanced to explain recovery from brain damage (Meyer, 1973).

\section{REFERENCES}

Altman, J. Effects of lesions in central nervous visual structures on light aversion in rats. American Journal of Physiology, $1962,202,1208$.

Horel, J. A., Bettinger, L. A., Royce, J. G., \& Meyer, D. R. Role of neocortex in the learning and relearning of two visual habits by the rat. Journal of Comparative and Physiological Psychology, 1966, 61, 66-78.

Meyer, P. M. Recovery from neocortical damage. In G. M. French (Ed.), Cortical functioning in behavior. Glenview, Illinois: Foresman, 1973.

Parker, T. D., Erickson, C. E., \& Treichler, F. R. The influence of brightness preferences on the assessment of cortical lesion 
deficits in rats. Journal of Comparative and Physiological Psychology, 1969,67, 240-244.

Parker, T. D., \& Treichler, F. R. The influence of restricted posterior neodecortication on brightness preference and brightness discrimination performance of rats. Physiology and Behavior, 1973, 10, 473-478.

Stratton, L. O., \& Edwards, S. Preference and sensory factors in brightness discrimination learning of posterior neodecorticated albino rats. Psychonomic Science, 1971.24, 9-10.
Thompson, R., \& Malin, C. F. The effect of neocortical lesions on retention of a successive brightness discrimination in rats. Journal of Comparative and Physiological Psychology, 1961, 54, 326-328.

(Received for publication August 20, 1974; revision received October 9,1974 .) 